\title{
La percepción de los asesores de formación permanente de TIC sobre la formación del profesorado
}

\author{
Ricardo Luengo Gonzálezi, Antonio Paniagua Navarroii, \\ José Luis Torres Carvalhoiii \& Luis M. Casas Garcíaiv \\ Universidad de Extremadura, España
}

\begin{abstract}
Resumen
La innovación en la práctica docente forma parte de la esencia de los modelos de formación del profesorado actuales. La revisión permanente de la práctica docente persigue mejorar los procesos de enseñanza/aprendizaje y avanzar hacia el éxito escolar formando a personas autónomas y capaces de ejercer una ciudadanía responsable. En este contexto, nos preguntamos qué opinión tienen los gestores de la formación sobre los cambios que se están produciendo en las diferentes modalidades formativas y en qué medida favorecen la consecución de los objetivos del sistema educativo. Este artículo resume el proceso seguido para conocer la postura de un grupo de asesores sobre una tendencia formativa. Para lograrlo de una forma abierta se empleó una aproximación cualitativa. La conclusión principal es que los asesores encuentran una gran utilidad a esta modalidad y que se debe profundizar en sus posibilidades.
\end{abstract}

Palabras-clave

Metodología cualitativa; Formación del profesorado; Innovación; TIC

\section{Marco Teórico}

Desde el comienzo del siglo XXI estamos asistiendo a los procesos de cambio en los que se encuentran inmersos tanto el sistema educativo español como los diferentes sistemas europeos. En esta permanente evolución, el profesorado requiere una formación permanente acorde con sus necesidades 
profesionales y personales. Como indicado por UNESCO (2011), las competencias profesionales se encuentran en constante construcción y todo aquello que se aprendió hace diez o quince años pierde vigencia para dejar paso a nuevas formas de enseñar y de aprender.

Uno de los cambios que nos trae la sociedad de la información y del conocimiento es la incorporación de los medios informáticos a nuestra vida diaria, tanto personal como profesional. Así, en el ámbito docente, la formación del profesorado que tradicionalmente se venía realizando de forma presencial ha incorporado con cierta naturalidad el cambio hacia una formación a distancia, empleando los avances informáticos a nuestro alcance. Del mismo modo, la formación del profesorado hacia una formación a distancia que complementa la formación presencial, como establecen diferentes autores como Prensky (2001) o Makri, Papanikolaou, Tsakiri, y Karkanis (2014).

Tanto las ventajas como los inconvenientes de la formación a distancia están enunciadas en el estudio realizado por Paniagua (2014) y son conocidas en su mayoría por el colectivo docente desde hace tiempo. Es una modalidad que se utiliza de forma generalizada aunque se complementa con los métodos tradicionales. Con este punto de partida se buscan formas de aprender que sean capaces de unir lo mejor de las dos posibilidades. Una de las más destacadas en los últimos años es blended learning, que combina fases de formación presencial con módulos formativos a distancia, tal y como expone Černá (2009). En esa modalidad hemos centrado nuestro esfuerzo, intentando determinar cuál sería la mejor fórmula para trasladar a la formación del profesorado un modelo mixto de trabajo.

La importancia de nuevos modelos de formación queda de manifiesto en estudios recientes, como destacan Linhares y Chagas (2014) al indicar que: "Los cursos tradiciones y su modelo de aprendizaje están siendo desafiados por comunidades y redes que ofrecen soluciones más eficaces para atender las demandas de las características variadas del proceso de aprendizaje" (p. 63, nuestra traducción). Igualmente destacan los autores en su relación entre diferentes modalidades: "(...) mientras que básicamente cuatro años atrás, lo online era un complemento para las reuniones presenciales de los grupos de estudio, hoy lo presencial pasa a ser un complemento en el que el contacto online es mayor que lo presencial (...)" (p. 66 , nuestra traducción). Si bien es una aseveración algo ambiciosa, es una tendencia a tener en cuenta. 
El objetivo principal de nuestra investigación consistió en determinar cómo puede aplicarse la modalidad blended learning en la formación del colectivo de docentes de niveles no universitarios. Como objetivo complementario, pretendimos comprobar si era posible sensibilizar a los asesores encargados de la formación sobre estas nuevas tendencias. Por último, se buscaban propuestas reales de implantación de esta metodología. Tanto en el proceso de sensibilización la que hacemos referencia como en las propuestas de implantación se buscaba conformar una imagen general de la aplicación de la modalidad formativa, lo que definimos como pregunta de investigación 1 .

El contexto de fondo de esta investigación, en el largo plazo, se haya en la búsqueda de un proceso de cambio efectivo y útil en la formación del profesorado. Si estamos de acuerdo en que las metodologías de trabajo en el aula y la relación entre docente y alumno están en fase de cambio, cabría preguntarse por qué no está produciendo de forma más palpable esa misma revisión de modelos en la metodología de formación del profesorado. Esta primera parte provoca esa primera reflexión por parte de las personas con el que se ha trabajado, mostrando una modalidad formativa que, si bien está extendida y es aparentemente conocida por todos, no tiene una aplicación excesivamente amplia en este ámbito concreto. ¿Cuáles son las razones? ¿Falta de utilidad? ¿Desconocimiento? ¿Resistencia al campo? Son esos interrogantes los que pretendemos desgranar en este proceso para decidir si es una buena vía a seguir.

El colectivo en el que se basó el trabajo se componía de un significativo grupo de asesores de formación permanente de la comunidad de Extremadura, en España (12 asesores participantes en el estudio, en un total de 18 asesores en Extremadura). Estas personas son docentes, como indica Nuñez de Lemus (2005), cuyo puesto de trabajo se renueva anualmente en los llamados Centros de Profesores y de Recursos, con el objetivo de gestionar las actividades formativas que se realizarán con el profesorado de su zona de influencia, además de facilitar asesoramiento y recursos para mejorar su desempeño. Entre las modalidades formativas que se desarrollan con normalidad encontramos cursos, grupos de trabajo, seminarios, cursos a distancia, etc. Cada modalidad tiene sus características específicas y persigue un fin concreto. Los asesores de formación permanente diseñan 
esas actividades, su temática y realizan un seguimiento completo de cada acción hasta su cierre final. En esta faceta de su trabajo, su labor es más próxima a la de la gestión de la formación que a lo entendido por un proceso de asesoramiento. Por ese motivo, en el informe se emplea indistintamente el término asesor y gestor para referirnos a las personas que han colaborado en el estudio.

Respecto a la metodología en estudio, el término blended learning se puede traducir a nuestro idioma como aprendizaje híbrido o aprendizaje combinado, aportando una idea clara de su significado. Las tres definiciones más habituales empleadas, de acuerdo con Graham (2006), por diferentes autores son:

- Combinar modalidades formativas (o distribución de recursos).

- Combinación de métodos formativos.

- Combinación de formación presencial y a distancia.

La fecha de aparición, hacia 2002, se vincula claramente con uno de sus rasgos más característicos, es el que establece la tercera definición, el de mezclar la tradición presencial con la formación a distancia. Otra característica, como pone de manifiesto Graham (2006), es que surge para unir dos mundos tradicionalmente separados, como es la formación presencial y a distancia.

Por tanto, el aprendizaje híbrido o blended learning no es más que la combinación de modalidades formativas y la acomodación de métodos de trabajo tradicionales con sistemas de formación a distancia, generando una nueva forma de aprendizaje que se ajusta a las posibilidades con las que contamos hoy en día. Es un sistema más acorde con las tendencias sociales de distribución de tiempo, espacio y relaciones interpersonales.

Coulon (2009) lo resume como una adaptación del modelo de aprendizaje a distancia destinado a facilitar a los estudiantes una combinación de canales para acceder al aprendizaje. Puede ser tan sencillo como una mezcla de sesiones presenciales con sesiones de tutores a distancia lo que lo ha popularizado pero, al mismo tiempo, puede ser un método extremadamente complejo, como definen Garrison y Kanuka (2004). Un diseño de blended learning representa una clara separación de la enseñanza presencial y de la enseñanza a distancia. Se trata de una reorganización de 
las dinámicas de enseñanza/aprendizaje comenzando con algunas necesidades contextuales y contingencias (disciplina, nivel de desarrollo y recursos). No existen dos diseños iguales de blended learning. Esto hace que la modalidad cuente con una gran complejidad. Por tanto, cada modelo requiere un estudio muy preciso y una adecuada planificación.

Otros autores, como Lim, Morris, y Kupritz (2007), se refieren a las diferentes variaciones que esta modalidad puede tener y validan la utilidad y actualidad de esta modalidad como una apuesta en auge, con su íntima relación con la modalidad a distancia. El resto de los estudios recientes revisados, como Bazhenov y Luchaninov (2014) o Sánchez y Arrufat (2015), trabajan en el mismo eje de investigación de diferentes formas de aplicar esta metodología, lo que demuestra su interés y el margen de aplicación tan amplio que aún presenta. Los estudios anteriores, más centrados en los estudiantes y en su aplicación en la enseñanza superior, constatan una mejora del interés de los usuarios, así como una buena predisposición a este cambio. Destacan igualmente, como elemento relevante, que esta modalidad favorece el aprendizaje autónomo en mayor medida que la enseñanza presencial.

\section{Método}

La intención de recoger un relato lo más amplio posible de las opiniones e ideas de los asesores TIC de Extremadura sugería que una aproximación interpretativa podría ser la más adecuada para el estudio, ya que el conjunto de elementos que enmarcan el abordaje de la metodología cualitativa se constituye en un proceso capaz de organizar, orientar y sistematizar el análisis e interpretación de los datos recogidos en la investigación, tal y como afirmaban Taylor y Bogdan (1987).

Debido al hecho de que es una metodología de investigación abierta, no resulta complicado localizar metodologías de trabajo estandarizadas que garanticen un buen enfoque funcional. En este caso, se empleó el esquema que Miles y Huberman (1994) definieron y que clarifica notablemente el proceso de recogida y análisis de datos cuantitativos. Se puede resumir en la siguiente figura, de acuerdo con Luengo y Casas (2013). 


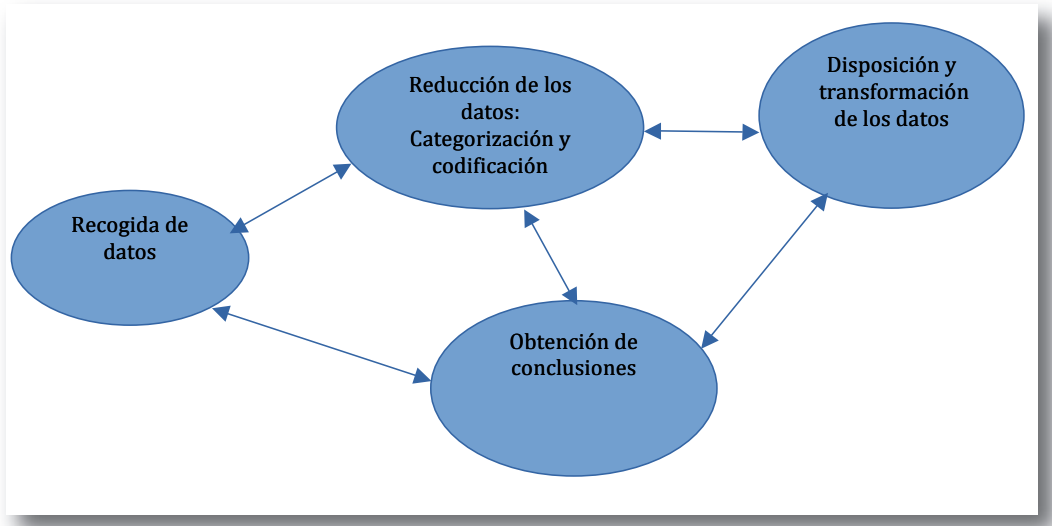

Figura 1 - El proceso de análisis de datos cualitativos

De este modo, se comienza el trabajo mediante la correspondiente fase de reflexión y de revisión de literatura existente, hasta llegar a la definición de los objetivos de la investigación, para concretar y describir las actividades que se desarrollan, como indican Cubo Delgado y Luengo González (2011), cuyas pautas han sido seguidas en gran medida en esta investigación: generación de hipótesis, definición operativa de variables y selección de instrumentos y del método de recogida de datos.

La definición de variables de la investigación resultó de gran utilidad, ya que tras esta importante tarea se pudo articular con facilidad el manejo, organización y análisis de datos a través del software de apoyo a la investigación cualitativa utilizado. Las variables que surgieron quedan reflejadas en el pequeño mapa conceptual de la figura 2, en el esquema que recoge el sistema de formación identificado y su relación con las variables detectadas.

Nuestra investigación contemplaría aspectos relacionados con las modalidades formativas (V1), con los asesores de formación (V2), con el profesorado (V3), con las herramientas empleadas (V4), y con cómo afectaban estas variables en el alumnado (V5). Esta última variable convertía el estudio en un proceso demasiado amplio y se optó por no incidir en exceso en ella para no distraer las conclusiones sobre lo que nos revelaba la primera 
variable. Por este motivo, se descartó en la fase de diseño del proceso de recogida de datos y no se incorporó al conjunto de cuestiones planteadas. Estas cuestiones se planificaron sobre esas variables y quedaron reflejadas en un modelo de base centrado en las cuatro primeras variables:

- V1. Modalidades formativas: ¿Qué ventajas e inconvenientes detectan en las diferentes modalidades? ¿Qué opinan sobre la formación a distancia? ¿Y sobre blended learning?

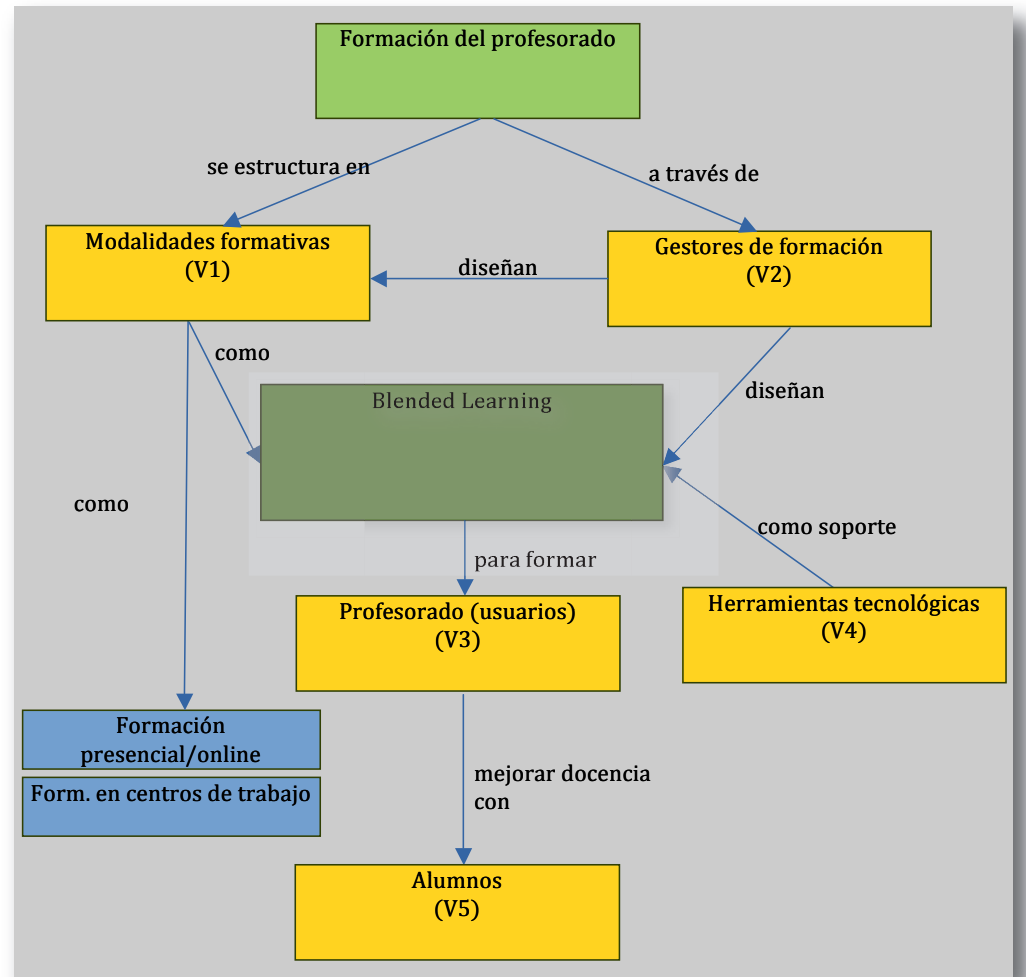

Figura 2 - Mapa conceptual de la investigación y su relación con el sistema analizado

- V2. Gestores de formación: ¿Se debe profundizar sobre nuevos modelos de formación? ¿Detectan innovación en este ámbito? 
¿Han cambiado sus prácticas de diseño de acciones en los últimos años? ¿Qué aspectos organizativos deben cambiar?

- V3. Profesorado: ¿Qué modalidades demanda el profesorado actualmente? ¿Qué características debe tener una actividad para que resulte atractiva al profesorado?

- V4. Herramientas tecnológicas: ¿Qué herramientas emplearían para diseñar una actividad basada en blended learning?

El proceso de recogida de datos que sigue a la definición de la investigación se centró en el uso de grupos focales online basados en Google Groups, atendiendo a las ventajas que aporta, entre ellas el modelo de participación poco intrusivo, como resume Edmunds (1999), y muy apropiado para abarcar participantes con las características del grupo de profesionales sobre el que se realiza el trabajo: disparidad de horarios, poco tiempo disponible para encuentros presenciales, gran dispersión geográfica (con distancias de más de 400 kilómetros entre los informantes, en los casos más extremos).

El diseño de los grupos online siguió en gran medida las propuestas formuladas por Stewart y Williams (2005) que distinguen entre grupos síncronos o asíncronos, siendo esta segunda opción la eficaz para este trabajo, al permitir que cada usuario estableciese sus tiempos de participación. Como los miembros del grupo de informantes son bastantes afines, se conocen hace tiempo y mantienen relaciones de trabajo frecuentes, no estaba en risco la ausencia de contribuciones al debate ni tampoco el aparecimiento de intervenciones minimalistas.

Además, se optó por realizar una división en dos grupos de seis participantes cada uno para facilitar las interacciones y el debate y para que fuese posible contrastar posteriormente sus respuestas. Un trabajo con los doce asesores en el mismo espacio podría haber provocado la inhibición de alguno de los participantes y dificultado la dinámica grupal de discusiones.

El trabajo de moderación que acompaña a todo el grupo focal estaba perfectamente definido de antemano y, genéricamente, se resumía a distribuir periódicamente las preguntas clave siguiendo un calendario preestablecido, a recentrar el debate y a efectuar aproximaciones a cada variable en estudio siempre que los participantes se dispersaban y a encontrar consensos dentro de cada grupo. 
Como soporte central del trabajo se diseño un conjunto de trece cuestiones abiertas destinadas a establecer un debate en cada uno de los dos grupos diferenciados que se crearon. Esas cuestiones fueron vinculadas cuidadosamente con las variables de la investigación, para asegurar que todas eran relevantes para el proceso. Igualmente las trece preguntas se distribuyeron en dos grupos de siete, repitiendo varias de ellas. Cada conjunto se utilizó con cada grupo, de tal modo que unos recibieron algunas preguntas diferentes a los otros.

El conjunto de las siete cuestiones de cada grupo focal comenzaba con unas preguntas introductorias sobre las modalidades formativas, comunes a ambos grupos, que pretendían provocar un debate en el que los participantes reflexionasen sobre su día a día laboral. Por ejemplo:

Cuestión 2. Ventajas de modalidades formativas.

Seguimos reflexionando sobre las ventajas claves de la modalidad formativa presencial y a distancia. Indica algunas de las ventajas que asocies a cada modalidad. Además, ¿crees que son compatibles?

Cuestión 3. Interés del profesorado.

En tu opinión, por lo que detectas del contacto con los usuarios, ¿cuáles son las modalidades formativas que más interesan al profesorado? ¿Por qué crees que es así?

A partir de la cuarta cuestión las propuestas se distanciaban. En el primer grupo el debate se mantenía en el ámbito del trabajo con modalidades formativas innovadoras, sin nombrar la temática del estudio abiertamente. Por el contrario, en el segundo caso se definió de forma más precisa la modalidad que nos interesaba conocer mejor, con cuestiones como estas:

Cuestión 5. Blended learning.

Blended learning, explicado en pocas palabras, consiste en generar acciones formativas que combinen diferentes formas de aprendizaje presencial con aprendizaje a distancia. ¿Qué te parece el concepto? ¿Qué ventajas crees que aporta respecto al resto de modalidades?

Cuestión 9. Organización en blended learning.

¿Qué aspectos organizativos sería necesario modificar en el diseño administrativo de una actividad basada en blended learning? ¿Y en el diseño de sus contenidos? 
Las últimas cuestiones de cada grupo se centraban ya en otros aspectos específicos, como posibles herramientas a utilizar, o la necesidad de profundizar en nuevas modalidades como agentes dinamizadores y responsables de la formación del profesorado en sus Centros de Formación de Profesorado.

Con el conjunto de cuestiones ya diseñado, los grupos comenzaron una singladura en la que en puntos preestablecidos se les iban proponiendo las diferentes cuestiones. El objetivo era dar flexibilidad a los debates mientras evitábamos que los grupos se quedasen en silencio. El proceso completo, desde los mensajes de bienvenida hasta la despedida, se extendió durante tres semanas.

Todo el proceso de preparación, así como las fases de análisis, quedaron reflejados en el siguiente esquema (Figura 3):

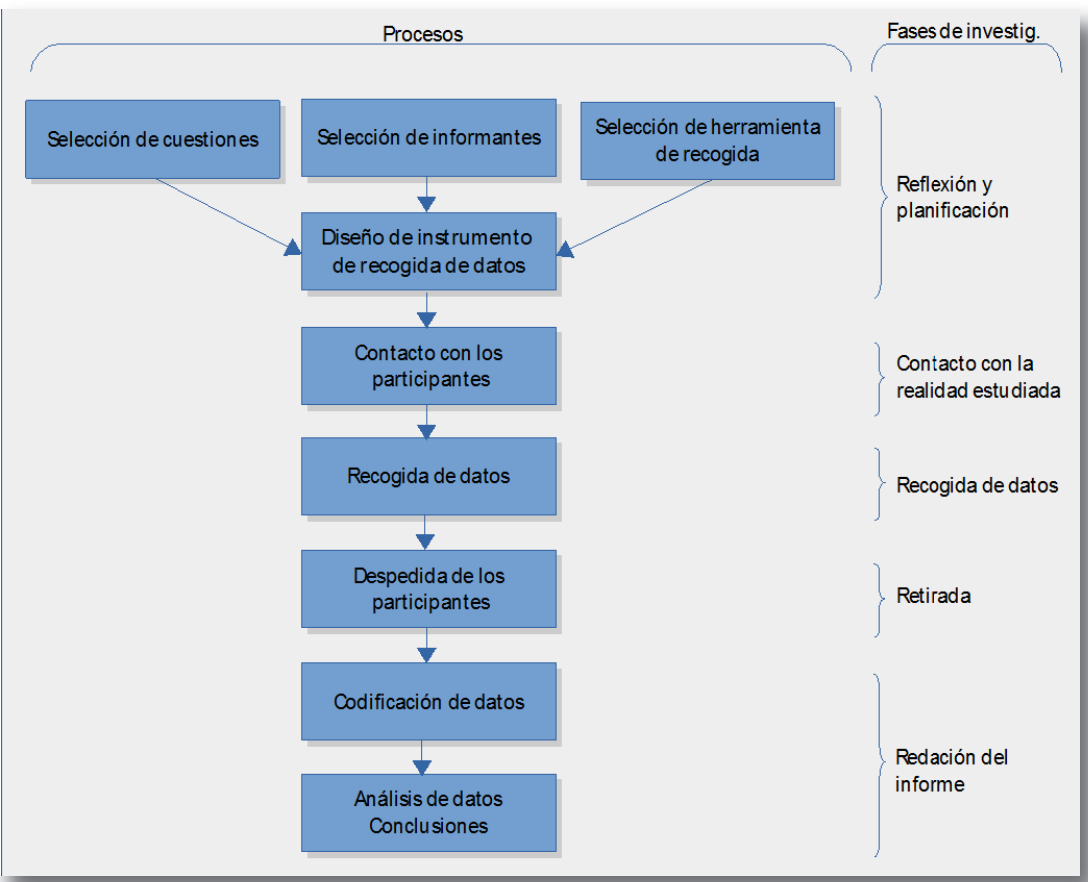

Figura 3 - Proceso de investigación 


\section{Recogida y codificación de los datos}

El gran problema al que se suele enfrentar una investigación basada en la metodología cualitativa es el de cómo convertir todos los datos consultados en uno conjunto organizado de información que permita una toma de decisiones clara y un análisis adecuado.

En nuestro estudio comenzamos el análisis con un proceso de conversión y extracción de datos. Desde los grupos focales online se procede a extraer los contenidos creados y a añadirlos en un formato que pueda ser recogido mediante un software de apoyo al análisis cualitativo de datos. En el estudio utilizamos WebQDA (Web Qualitative Data Analysis) que, según Souza, Costa, y Moreira (2011), es una herramienta de análisis basada en tecnología web, que facilita el trabajo colaborativo de investigadores y que sirve para editar, visualizar, asociar y organizar fuentes textuales y multimedia. Permite, además, crear categorías, codificar, controlar, filtrar, hacer búsquedas y consultas a los datos para responder a las preguntas de investigación.

En ese proceso se modifican los nombres de los participantes para mantener su anonimato, así como las referencias a sus centros de trabajo. Se utiliza una codificación sencilla: el moderador aparece expresado con la letra $\mathrm{M}$; los participantes se indican mediante un código Ax o Bx, dependiendo del grupo.

Tras realizar un tratamiento de la información y conversión a texto se produce la creación de un conjunto de nodos o categorías que recogerán las frases o palabras relevantes para la investigación. Como fruto de esta fase se obtuvieron cinco nodos de primer nivel, que se correspondían con las cuatro principales variables del estudio más una que surgió por sus constantes referencias por parte de los participantes, como era la administración educativa. De esos nodos se generaron diferentes subdivisiones dependiendo del tipo de respuesta que iban proporcionando los participantes o por la categorización de determinados aspectos, como en el caso de las modalidades formativas.

\section{Estudio y análisis de los datos}

El estudio de los datos obtenidos sigue el transcurso natural de una metodología cualitativa. Como punto de partida generamos una serie de 
sencillas tablas de frecuencia que nos permitían ver los términos más empleados en cada uno de los dos grupos. Los términos que más destacan, una vez obviadas las palabras más comunes, son 'horas', 'tiempo', 'trabajo', 'actividad', 'profesorado', 'ventajas' e 'inconvenientes'. Todas ellas dan una idea de la preocupación de los asesores de formación hacia materias como la duración de las modalidades formativas, el esfuerzo requerido en su ejecución y la relevancia para el profesorado. La lectura de las intervenciones, en efecto, releva esas cuestiones de forma recurrente. La tabla 1 muestra los términos más frecuentes.

\begin{tabular}{|l|c|}
\hline Palabra & Repetición \\
\hline Formación & 152 \\
\hline Distancia & 96 \\
\hline Presencial & 80 \\
\hline Cursos & 69 \\
\hline Curso & 66 \\
\hline Modalidad & 65 \\
\hline Formativas & 54 \\
\hline Modalidades & 53 \\
\hline Aprendizaje & 42 \\
\hline Trabajo & 40 \\
\hline Presenciales & 39 \\
\hline Profesorado & 39 \\
\hline Inconvenientes & 36 \\
\hline Horas & 35 \\
\hline
\end{tabular}

Tabla 1 - Términos más frecuentes

A continuación generamos una serie de matrices bidimensionales que relacionaban las variables del estudio con las partes del debate en la que se tocaban. Esto nos permitió detectar patrones concretos, como casos en los que determinadas variables aparecían constantemente en diferentes lugares sin que se hubiesen sugerido. Así, por ejemplo, se detecta en el estudio que la modalidad de blended learning, formulada como tal, no podría llegar a ejecutarse sin un consenso o autorización previa por parte de la administración educativa. Cada vez que se hablaba de innovación en los dos grupos o de aplicar cambios en las modalidades formativas, aparecía la administración como un obstáculo casi insalvable. Esto podría mostrar una relación compleja entre los asesores y el sistema que los organiza, donde no parece haber espacio a innovaciones que surgen de la base, de los centros o de los propios docentes. La tabla 2 recoge las referencias a la citada variable en las diferentes cuestiones. 


\begin{tabular}{|c|c|c|c|c|}
\hline Matriz & Herramientas & $\begin{array}{l}\text { Modalidades } \\
\text { formativas }\end{array}$ & $\begin{array}{l}\text { Gestores de } \\
\text { formación }\end{array}$ & Profesorado \\
\hline \multicolumn{5}{|l|}{ Grupo 1} \\
\hline $\begin{array}{l}\text { Cuestión } 1 \text {. Inconvenientes de } \\
\text { modalidades formativas. }\end{array}$ & $x$ & $\mathrm{x}$ & $\mathrm{x}$ & $x$ \\
\hline $\begin{array}{l}\text { Cuestión } 2 \text {. Ventajas de modalidades } \\
\text { formativas. }\end{array}$ & & & $\mathrm{x}$ & $x$ \\
\hline Cuestión 3. Interés del profesorado. & & & $x$ & $x$ \\
\hline $\begin{array}{l}\text { Cuestión } 4 \text {. Innovación en las acciones } \\
\text { formativas. }\end{array}$ & & & $x$ & \\
\hline Cuestión 5. Seminarios virtuales. & & & $\mathrm{x}$ & $\mathrm{x}$ \\
\hline Cuestión 6. Modalidades mixtas. & & & $x$ & $x$ \\
\hline Cuestión 7. Herramientas. & $x$ & & & \\
\hline \multicolumn{5}{|l|}{ Grupo 2} \\
\hline $\begin{array}{l}\text { Cuestión } 1 \text {. Inconvenientes de } \\
\text { modalidades formativas. }\end{array}$ & $x$ & & $\mathrm{x}$ & \\
\hline $\begin{array}{l}\text { Cuestión 2. Ventajas de modalidades } \\
\text { formativas. }\end{array}$ & & & $x$ & \\
\hline Cuestión 3. Interés del profesorado. & & & $x$ & $x$ \\
\hline \multicolumn{5}{|l|}{ Cuestión 4. Blended learning. } \\
\hline \multicolumn{5}{|l|}{$\begin{array}{l}\text { Cuestión } 5 \text {. Características de blended } \\
\text { learning. }\end{array}$} \\
\hline $\begin{array}{l}\text { Cuestión } 6 \text {. Organización en blended } \\
\text { learning. }\end{array}$ & & & $\mathrm{x}$ & \\
\hline $\begin{array}{l}\text { Cuestión } 7 \text {. Interés de los gestores de } \\
\text { formación. }\end{array}$ & & & $x$ & $x$ \\
\hline
\end{tabular}

\section{Tabla 2 - Ocurrencia de la variable administración}

Resulta evidente que en el primer grupo se centraban más en los aspectos normativos que en el caso del segundo grupo, si bien la diferencia de ocurrencias no es concluyente.

Analizamos, además, cada una de las referencias que se hacían a las diferentes variables definidas usando los nodos que se detectaron en la fase de codificación de datos.

Se aprecia cierta facilidad para describir las modalidades más populares, tal y como se les solicitaba. Es destacable que en la modalidad similar a blended learning que ya están trabajando aparecen más inconvenientes que ventajas. Por otro lado, es notable también la abundancia de propuestas de formato para diseñar una modalidad de blended learning. 
En el transcurso de los debates se apreciaban muchas aportaciones en este sentido (Tabla 3).

\begin{tabular}{|c|c|c|c|c|}
\hline Variable & Concepto & & & Referencias \\
\hline \multirow{11}{*}{$\begin{array}{l}\text { Modalidades } \\
\text { formativas }\end{array}$} & \multirow{6}{*}{$\begin{array}{l}\text { Modalidades } \\
\text { clásicas }\end{array}$} & \multirow{2}{*}{ Curso } & Inconvenientes & 28 \\
\hline & & & Ventajas & 27 \\
\hline & & \multirow{2}{*}{ Curso a distancia } & Inconvenientes & 30 \\
\hline & & & Ventajas & 27 \\
\hline & & \multirow{2}{*}{ Formación en centro } & Inconvenientes & 1 \\
\hline & & & Ventajas & 7 \\
\hline & & \multirow{3}{*}{ Blended Learning } & Inconvenientes & 4 \\
\hline & \multirow{4}{*}{$\begin{array}{l}\text { Modalidades } \\
\text { modernas }\end{array}$} & & Ventajas & 9 \\
\hline & & & Formato & 33 \\
\hline & & \multirow{2}{*}{ Seminarios virtuales } & Inconvenientes & 7 \\
\hline & & & Ventajas & 4 \\
\hline
\end{tabular}

Tabla 3 - Variable 1: Modalidades formativas

A la vista de los comentarios de los asesores sobre su propia práctica, nos pareció conveniente destacar cómo junto a la problemática que encontraban, muy abundante, el número de soluciones era igualmente alto, así como propuestas de mejora e innovación en su trabajo (Tabla 4).

\begin{tabular}{|lll|}
\hline Variable & Concepto & Referencias \\
\hline \multirow{2}{*}{$\begin{array}{l}\text { Gestores de } \\
\text { formación }\end{array}$} & Problemas & Soluciones \\
& Innovación & 23 \\
& & 27 \\
\hline
\end{tabular}

\section{Tabla 4 - Variable 2: Gestores de formación}

El profesorado no ha sido en todas las respuestas el centro de atención, si bien surge recurrentemente. Aquí prevalece la problemática endémica de la formación del profesorado. Se recoge también con bastante fidelidad las preferencias en lo que se refiere a la formación por parte del profesorado desde la óptica del formador (Tabla 5). 


\begin{tabular}{|lll|}
\hline Variable & Concepto & Referencias \\
\hline \multirow{3}{*}{ Profesorado } & Problemas & Soluciones \\
& Preferencias & 19 \\
& & 5 \\
\hline
\end{tabular}

Tabla 5 - Variable 3: Profesorado

En la variable Herramientas (Tabla 6), se consideró interesante conocer el número de veces que citaba a Moodle como la base de un modelo de blended learning. Se produjo un número significativo, dado que el objetivo no era incidir en exceso en la temática de las herramientas TIC, sino en la metodología.

\begin{tabular}{|lll|}
\hline Variable & Concepto & Referencias \\
\hline \multirow{3}{*}{ Herramientas } & Moodle & 3 \\
& Herramientas informáticas & 17 \\
\cline { 2 - 3 } & Medios & 9 \\
\hline
\end{tabular}

\section{Tabla 6 - Variable 4: Herramientas}

\section{Resultados}

Mediante las tablas definidas se pueden constatar los elementos fundamentales que han ido destacando los grupos en relación con cada variable. De ello se extraen algunas conclusiones:

- El grupo conoce las modalidades formativas y establece con facilidad sus ventajas e inconvenientes.

- Detectan los problemas frecuentes de su trabajo y las soluciones a los mismos, además de proponer innovaciones.

- El profesorado no es exclusivamente el centro de su trabajo, aunque se les menciona de forma recurrente.

- Identifican las herramientas necesarias para implementar modalidades formativas innovadoras.

- La administración educativa tiene una frecuencia recurrente en sus debates.

Podemos sacar conclusiones detalladas como fruto del estudio para comprobar las respuestas dadas y cotejarlas con el resto de los estudios que habíamos revisado en el marco teórico del trabajo (Paniagua, 2014). 


\section{Modalidades formativas}

En el ámbito de las modalidades formativas, los asesores de formación permanente detectan correctamente los problemas asociados a la formación presencial:

- Carga horaria y desplazamientos.

- Formación en horario de tarde.

- Conciliación familiar.

- Rigidez en el modelo.

Mientras que sus ventajas en este sentido son:

- Dinamismo en el proceso.

- Aprendizaje en grupo.

- Acceso directo a un experto.

En el caso de la formación a distancia, también coinciden en sus inconvenientes:

- Dificultad de uso de las TIC.

- Exceso de tiempo autónomo.

- Soledad y falta de relación.

Y en la identificación de sus ventajas:

- Ahorro de tiempo.

- Facilidad de organización.

- Ahorro en desplazamientos.

- Menor coste.

\section{Blended Learning}

Sobre la temática central del estudio, el aprendizaje combinado o blended learning, encontramos dos posturas:

- La primera tendencia es la necesidad de ajustes normativos para que se contemple esta modalidad. Este es un obstáculo insuperable para el gestor de formación, ya que no tiene otra posibilidad más que sugerir los cambios. 
- La segunda tendencia es la de la incomprensión por parte del profesorado de la filosofía de la modalidad.

Sobre sus ventajas, el número de referencias es mayor, posiblemente por la reflexión previa. Hay una cierta coincidencia en que resolvería los inconvenientes de las modalidades anteriores. Lo resume este comentario:

(...) con la combinación de ambas modalidades aprovechamos lo que la tecnología nos ofrece y no deshumanizamos el proceso formativo, tendríamos las ventajas de ambas modalidades y eliminamos algunos de sus inconvenientes. (Part. B5)

\section{Gestores de formación}

Al analizar su propia práctica como asesores de formación en su faceta más administrativa, de gestión de las actividades, los participantes demuestran un gran conocimiento de sus dinámicas de trabajo y llegan a conclusiones interesantes, como su capacidad de innovación en relación con otras variables. Queda reflejado en esta intervención:

Yo veo mejoras y novedades en la manera en que nos comunicamos con los docentes, en la inmediatez con que se divulgan las convocatorias, en la inclusión de alguna modalidad formativa (oficial o extraoficialmente), en la manera en que se coordina la red de formación, en el grado de colaboración entre los CPR o la gestión del trabajo en los equipos pedagógicos... (...) Por supuesto que la normativa siempre irá detrás de las necesidades, pero creo que el impulso innovador debe venir de "abajo" hacia "arriba", haciendo propuestas realistas y eficaces. (Part. A7)

Es una conclusión casi unánime. La regulación y la normativa alumbra buena parte de su trabajo diario.

Un detalle clave es cómo organizar una modalidad formativa que combine lo presencial con lo no presencial de forma correcta. El análisis de los datos nos muestra una gran cantidad de propuestas organizativas (hasta 33 referencias) para implementar esta modalidad. Éstas van desde detalles como la ubicación o el coste económico hasta la más repetida, la distribución horaria del tiempo. En este sentido, en el debate se llega a la conclusión de que la fase presencial de una actividad blended learning debería rondar el $25 \%$ de la duración de la misma. 
En otros casos, las intervenciones son menos explícitas y se centran en los aspectos más generales de la modalidad, coincidiendo con los diferentes autores revisados en el marco teórico. Por ejemplo:

(...) b-learning ofrece posibilidades muy interesantes, siempre que sepamos combinar las sesiones presenciales y no presenciales de forma adecuada, atendiendo a las necesidades reales de los participantes e intentando que los grupos sean lo más homogéneos posible, aunque esto último es algo muy difícil de lograr en la formación permanente, a no ser que se afine mucho en los criterios de selección (otro tema que me parece clave para que la relación modalidad/interés sea óptima. (Part. A7)

También resulta destacable el hecho de que en muy pocos momentos se ha hecho referencia al proceso de evaluación inherente a estas modalidades formativas. Ese es un aspecto muy relevante en su trabajo pero del que parecen haberse olvidado en su relato, posiblemente al dar por sentado que se trataría de asistir a todas las sesiones presenciales y realizar algunas tareas online, como vienen realizando en las aproximaciones a esta modalidad.

\section{Profesorado}

El profesorado atrae parte de los comentarios expresados por los asesores, con ideas relevantes como:

Esto no quiere decir que muchos vienen a los cursos a aprender cómo mejorar técnicas metodológicas de aula, conocimientos TIC, etc. Hay que diferenciar entre los cursos que tienen que hacer y los cursos que les gusta hacer. (Part. A1)

Mientras que en otros casos se indica la necesidad de todas las modalidades:

Pienso que ningún profesor se inclina de manera única por un tipo de formación, sino que se busca combinar las dos. (Part. B6)

Otro aspecto que se vincula al profesorado es el equilibrio que se debe seguir para que las actividades atraigan al profesorado, llegando a una actividad modelo de formación en esta temática que rondaría las 100 horas de duración, con 25 de ellas presenciales. 


\section{Herramientas tecnológicas}

Sobre las herramientas tecnológicas, los asesores consideran que un buen soporte para la parte no presencial sería el uso de Moodle, que es el sistema que ya emplean de forma habitual, si bien se atreven a aventurar otras alternativas. Veamos este ejemplo, donde se aprecia también la reflexión realizada por los participantes:

Yo también apostaría Moodle, complementadolo con muchas de las herramientas que habéis citado e integrando en la medida de lo posible los contenidos en el curso elaborados desde otras aplicaciones gracias al formato SCORM, utilizando videconferencias con BigBlueButton, talleres con evaluación por rúbricas... Las posibilidades de Moodle son enormes y creo que normalmente no las aprovechamos lo suficiente. (Part. A5)

\section{Discusión de resultados}

Aunque en diferentes apartados ya dejamos ver las analogías con otros autores, los resultados obtenidos confirman que son consistentes con la literatura consultada. El colectivo estudiado coincide en valorar positivamente las metodologías estudiadas, como indicaban Makri (2014) o Černá (2009), y detectan las mismas fortalezas y debilidades que aparecen en este estudio, así como los matices organizativos contemplados.

Como contraste con otros autores, el colectivo no ha destacado el uso de diferentes tipologías de actividades presenciales o a distancia dentro del bloque de la modalidad blended learning, como destacan Lim et al. (2007) y otros autores, que analizan varios formatos diferentes. Se percibe que los asesores, seguramente por la falta de aplicaciones más específicas de la modalidad, han dado por sentado, desde un primer momento, que se trata de realizar una parte de formación específica a distancia y una parte presencial expositiva, con un ponente que transmite información a los usuarios.

Otra diferencia con lo observado por autores como Bazhenov y Luchaninov (2014) es la escasa referencia al proceso de evaluación y certificación de las actividades basadas en la modalidad blended learning.

En lo referente a los aspectos metodológicos del estudio, se ha podido constatar la existencia de las mismas dificultades y precauciones de los autores consultados a la hora de diseñar los grupos focales. El modelo no intrusivo desarrollado (Edmunds, 1999) simplificó el esfuerzo de los 
participantes, atendiendo a sus horarios y a su distancia geográfica. Por su parte, el hecho de que el proceso se haya desarrollado como un grupo asíncrono (Stewart \& Williams, 2005) ha favorecido también la aparición de comentarios muy reflexivos y bien desarrollados. Como diferencia, los aspectos referentes a la dificultad de acceso a los medios informáticos no son ya tan relevantes, dado que este colectivo poseía un alto nivel de cualificación en ese aspecto. La precisión de que el trabajo requería el uso de un grupo focal en su modalidad online debe ser destacado de nuevo, dado que resultaba imposible realizar el trabajo de otra forma. A la vista de la información recabada podemos afirmar que ha sido una solución satisfactoria. No se desprende del análisis de datos la necesidad de haber contado con más datos. La decisión de dividir en dos grupos al colectivo ha permitido observar que no se producen diferencias relevantes y que las respuestas eran recurrentes en ambos casos, con mínimas diferencias.

\section{Conclusiones}

La conclusión general a la que llegamos es que los participantes en este estudio son grandes conocedores de las tendencias formativas y, a través de la práctica, alcanzan conclusiones similares a las de los estudios revisados en el marco teórico. Todos ellos consideran necesario profundizar en modalidades como blended learning, la consideran atractiva para el profesorado y son conscientes de las dificultades intrínsecas de su implantación. Reclaman igualmente un apoyo institucional para conseguirlo.

El objetivo de nuestra investigación, pues, consigue cumplirse en líneas generales. Es factible trabajar esta modalidad y, en este primer acercamiento a la cuestión, los agentes implicados son ya capaces de definir las fortalezas y debilidades del modelo, así como su modo de implantación. Los objetivos complementarios planteados también se han alcanzado, dado que con estos grupos hemos conseguido fomentar la reflexión de la práctica profesional de los participantes, hemos buscado propuestas específicas y hemos alcanzado un pequeño catálogo de aplicaciones útiles para el diseño y ejecución de actividades basadas en blended learning.

Como limitación de la investigación se pone de manifiesto que se debe profundizar en tanto en los aspectos relacionados con la evaluación y la 
certificación de actividades como en lo que se refiere a las tipologías internas del desarrollo de la modalidad que deberían ser fruto de estudio en posteriores trabajos. Son aspectos tratados en otros trabajos analizados que no parecen resultar relevantes para los asesores de formación permanente en su relación con esta modalidad, mientras que en el resto de modalidades sí, son muy importantes.

En los aspectos metodológicos cabe destacar que la realización de un estudio de estas características es compleja. Se parte de un grupo abierto, con poca disponibilidad y con un gran conocimiento de la materia a tratar. Ante estas premisas, el uso de una metodología cualitativa poco invasiva se convierte en la solución idónea.

Por último, como vías futuras de trabajo, consideramos que se puede seguir profundizando en formatos de implantación de modalidades blended learning en la formación del profesorado de niveles no universitario. Se requiere, además, una reinvención del formato para alejarlo de mezclas más o menos acertadas de la modalidad presencial y de la modalidad a distancia, para poder determinar si de ahí puede emanar un formato más acorde con las necesidades actuales.

\section{Referências}

Bazhenov, R. I., \& Luchaninov, D. V. (2014). Use of blended learning elements for formation of a humanitarian student's creative initiative at learning modern information technologies. Life Science Journal, 11(11s), 371-374.

Černá, M. (2009). Blended learning experience in teacher education: The trainees' perspective. Acta Didactica Napocensia, 2(1), 37-48.

Coulon, A. (2009). E-learning initiatives for teachers and trainers in the Mediterranean region: Comparative analyses. Torino: European Training Foundation.

Cubo Delgado, S., \& Luengo González, R. (2011). El proceso de planificación de la investigación. In S. Cubo Delgado, B. Martín Marín, \& J. L. García Ramos (Coords.), Métodos de investigación y análisis de datos en ciencias sociales y de la salud (pp. 45-89). Madrid: Ediciones Pirámide Grupo.

Edmunds, H. (1999). The focus group research handbook. Lincolnwood, III: NTC Business Books.

Garrison, D. R., \& Kanuka, H. (2004). Blended learning: Uncovering its transformative potential in higher education. The Internet and Higher Education, 7(2), 95-105. 
Graham, C. R. (2006). Blended learning systems. In C. J. Bonk \& C. R. Graham, The handbook of blended learning: Global perspectives, local designs. San Francisco, CA: Pfeiffer.

Lim, D. H., Morris, M. L., \& Kupritz, V. W. (2007). Online vs. blended learning: Differences in instructional outcomes and learner satisfaction. Journal of Asynchronous Learning Networks, 11(2), 27-42.

Linhares, R., \& Chagas, A. (2014). A contribuição do Facebook no processo de aprendizagem colaborativa/Facebook's contribution in the process of collaborative learning. In A. P. Costa, L. P. Reis, F. N. de Souza, \& R. Luengo (Eds.), Libro de Actas de $3^{\circ}$ Congreso Ibero-Americano en Investigación Cualitativa/ Livro de Atas do "3० Congresso Ibero-Americano em Investigação Qualitativa. Vol. I: Artículos de Educación/Artigos de Educação (pp. 62-67). Oliveira de Azeméis: Ludomedia.

Luengo, R., \& Casas, L. M. (2013). Documentación de la asignatura 'Diseño y Metodología de Investigación'. Extremadura: Universidad de Extremadura, Departamento de Didáctica de las CC Experimentales y de las Matemáticas.

Makri, K., Papanikolaou, K., Tsakiri, A., \& Karkanis, S. (2014). Blending the community of inquiry framework with learning by design: Towards a synthesis for blended learning in teacher training. Electronic Journal of e-Learning, 12, 183-194.

Miles, M. B., \& Huberman, A. M. (1994). Qualitative data analysis: An expanded sourcebook. Thousand Oaks, CA: Sage.

Nuñez de Lemus, A. (2005). La formación permanente del profesorado en Extremadura: Veinte años de centro de profesores y recursos. Revista de Estudios Extremeños, $n^{\circ}$ III, 1077-1108.

Paniagua, A. (2014). Tendencias en modalidades formativas. Blended learning (Trabajo Fin de Máster). Universidad de Extremadura, Departamento de Didáctica de las CC Experimentales y de las Matemáticas, Extremadura.

Prensky, M. (2001). Digital natives, digital immigrants - Part 1. On the horizon, 9(5), 16.

Sánchez, V. G., \& Arrufat, M. J. G. (2015). Modelo de análisis de metodologías didácticas semipresenciales blended learning en Educación Superior. Educación XX1, 19(1), 39-61.

Souza, F. N., Costa, A. P., \& Moreira, A. (2011). Análise de dados qualitativos suportada pelo software WebQDA. In P. Dias \& A. J. Osório (Orgs.), Atas da VII Conferência Internacional de TIC na Educação: Perspetivas de Inovação (pp. 49-56). Braga: Universidade do Minho.

Stewart, K., \& Williams, M. (2005). Researching online populations: The use of online focus groups for social research. Qualitative Research, 5(4), 395-416.

Taylor, S. J., \& Bogdan, R. (1987). Introducción a los métodos cualitativos de investigación. Buenos Aires: Paidós.

UNESCO (2011). UNESCO ICT competency framework for teachers (version 2.0). Paris: United Nations Educational, Scientific and Cultural Organization. 


\section{A PERCEÇÃO DOS ASSESSORES DE FORMAÇÃO CONTÍNUA EM TIC SOBRE A FORMAÇÃO DE PROFESSORES}

\section{Resumo}

Na prática docente, a inovação faz parte da essência dos atuais modelos de formação de professores. A atualização permanente da prática docente pretende melhorar os processos de ensino e aprendizagem e avançar no sentido do sucesso escolar, formando pessoas autónomas e capazes de exercer uma cidadania responsável. Neste contexto, pretendemos conhecer a opinião dos gestores da formação contínua de professores sobre as mudanças que se estão a produzir nas diferentes modalidades formativas e em que medida favorecem a consecução dos objetivos do sistema educativo. Este artigo resume o processo desenvolvido para conhecer a postura de um grupo de assessores sobre uma determinada tendência formativa. Para o conseguir, foi utilizada uma aproximação metodológica de natureza qualitativa. Como conclusão principal, destacamos que os assessores encontram uma grande utilidade nesta modalidade formativa e consideram que é importante prosseguir com a sua implementação.

Palavras-chave

Metodologia qualitativa; Formação contínua de professores; Inovação; TIC

\section{THE PERCEPTION OF ICT TRAINING ADVISORS ABOUT TEACHER TRAINING}

\section{Abstract}

Innovation in teaching is part of the essence of the current models of teacher training. The ongoing review of teaching practice aims to improve the 
teaching/learning process and to improve the success of the pupils, while fostering a responsible citizenship. In this context, we aim to know the opinion training advisors have about the changes that are occurring in the different training modalities and the extent to which they favor the achievement of the objectives of the educational system. This paper summarizes the process followed to know the impressions of a group of training advisors about a specific training trend. In order to achieve the aims in an open and unconditioned way, a qualitative approach has been used. Training advisors find this modality useful and they think it should be explored in the near future.

\section{Keywords}

Cualitative methodology; Teacher's training; Innovation; ICT

Recebido em dezembro/2015

Aceite para publicação em maio/2016

i Grupo de Investigación CIBERDIDACT, Departamento de Didáctica de las Ciencias Experimentales y de las Matemáticas, Facultad de Educación, Universidad de Extremadura, España.

ii Grupo de Investigación CIBERDIDACT, Facultad de Educación, Universidad de Extremadura, España.

iii Grupo de Investigación CIBERDIDACT, Departamento de Didáctica de las Ciencias Experimentales y de las Matemáticas, Facultad de Educación, Universidad de Extremadura, España.

iv Grupo de Investigación CIBERDIDACT, Departamento de Didáctica de las Ciencias Experimentales y de las Matemáticas, Facultad de Educación, Universidad de Extremadura, España.

Toda a correspondência relativa a este artigo deve ser enviada para: Ricardo Luengo González, Universidad de Extremadura - Facultad de Educación, Campus de Badajoz, Av. de Elvas, s/n, 06006 Badajoz, España. Email: rluengo@unex.es 